\title{
5GHz MMIC LNA Design Using Particle Swarm Optimization
}

\author{
${ }^{1}$ Low Wen Shin, *Neoh Siew Chin ${ }^{1}$, Arjuna Marzuki² \\ 1Universiti Malaysia Perlis, Malaysia \\ 2Universiti Sains Malaysia, Malaysia \\ *scneoh@unimap.edu.my
}

\begin{abstract}
This research presents an optimization study of a $5 \mathrm{GHz}$ Monolithic Microwave Integrated Circuit (MMIC) design using Particle Swarm Optimization (PSO). MMIC Low Noise Amplifier (LNA) is a type of integrated circuit device used to capture signal operating in the microwave frequency. This project consists of two stages: implementation of PSO using MATLAB and simulation of MMIC design using Advanced Design System (ADS). PSO model that mimics the biological swarm behavior is developed to optimize the MMIC design variables in order to achieve the required circuit performance and specifications such as power gain, noise figure, drain current and circuit stability factor. Simulation results show that the proposed MMIC design fulfills the circuit stability factor and achieves a power gain of $19.73 \mathrm{~dB}$, a noise figure of $1.15 \mathrm{~dB}$ and a current of $0.0467 \mathrm{~A}$.
\end{abstract}

Keywords: MMIC, low noise amplifier, PSO, optimization, parameter tuning

\section{Introduction}

Monolithic Microwave Integrated Circuit (MMIC) is a type of integrated circuit (IC) device that operates at microwave frequencies of $300 \mathrm{MHz}$ to $300 \mathrm{GHz}$. These devices are widely used for microwave mixing, power amplification, low noise amplification, and high frequency switching (Marsh, 2006; Robertson and Lucyszyn, 2001). This research focuses on the design of MMIC low noise amplifier (LNA). LNA is considered as an electronic amplifier used in communication systems, amplifying very weak antenna captured signals. Nowadays, Global Positioning System (GPS) is among the widely used microwave systems. LNA overcomes noise and reduces additional interference. According to (Lucek and Damen, 1999; Yang et al., 2009), power gain noise figure are crucial parameters for LNA design and nonlinear distortion will be produced if gain, stability and noise are not fulfilled by the designed LNA. In this project, PSO (Particle Swarm Optimization) is used to optimize the $5 \mathrm{GHz}$ MMIC LNA. PSO is one of the swarm intelligence techniques that are widely used to perform numerical optimization by simulating the social behavior of birds. The representation of the flocking behavior of birds was later being used in computer simulation of virtual birds, known as Boids. Knowing the suitability of the formulation of bird's behavior, the Particle Swarm Optimizer was derived (Kennedy \& Eberhart, 1995). In PSO, the representation of the optimization problem is similar to the encoding methods used in Genetic Algorithms (GAs). Instead of using genes and chromosomes in GAs, the variables in PSO are designated as particles with multi-dimensional hyperspace. These particles search through the hyperspace, finding the global minima/maxima with the movement governed by a mathematical equation. In this project, MATLAB is used to develop the PSO model for optimizing the MMIC LNA. On the other hand, circuit performance of the designed MMIC LNA is simulated using ADS, leading electronic design automation software for RF, microwave, and signal integrity applications. ADS is employed by many leaning industries due to the innovative and commercially successful technologies applicable to the application of wireless communication, networking and aerospace industries. In this research, MATLAB-based PSO integrates with the ADS circuit simulator to assist the design of the MMIC LNA. The proposed integration aims to fulfill the MMIC LNA design specifications and constraints in terms of power gain $(\mathrm{dB}(\mathrm{S}(2,1))$, noise figure (NFmin_out), drain current (ID), and two circuit stability factors (mu_load \& mu_source).

\section{MMIC LNA DESIGN}

In LNA design, the most important factors to be considered are low noise, moderate gain, matching and stability. LNAs are one of the key building blocks for RF receivers that play critical role in determining the overall system noise figure (NF) of the receiver (Tulunay \& Balkir, 2004). Conventionally, many LNA designs are carried out in a trial and error manner. Although several CMOS RF building blocks that achieved tough requirements have been recently realized [Gil, Han, \& Shin, 2003; Taris et al., 2003; 
Gatta et al., 2001], there is no systematic design methodology that accounts for many specifications. Thus, an optimal design is required for simultaneous consideration of all specifications. Both active and passive devices with parasitic elements should be taken into account during the design procedure to ensure design stability. From the literature review, optimization of RF ICs has been addressed by a number of researchers in the past (Gupta \& Allstot, 1998; Ge \& Mayaram, 1998; Nocedal \& Wright, 1999; Niknejad \& Meyer, 1998; Vancorenland et al., 2000). However, in accord to Tulunay \& Balkir (2004), many of the reported results cannot directly match the simulation results of commercially available CAD tools. As a result, an optimization model that could provide an accurate starting point and consequently minimize the design time in a professional RF CAD environment is very much desired.

This research focuses on the optimization of the MMIC LNA designed in (Neoh, et al., 2008). Neoh, et al. (2008) focuses on $3 \mathrm{GHz}$ MMIC LNA whereas this research focuses on a 5GHz MMIC LNA. The devised circuit consists of two stages RC feedback amplifier with transistors M1 and M2 of 50 $\mu$ m unit gate width. Figure 1 shows a schematic diagram of the designed circuit. From the figure, $\mathrm{C}_{\mathrm{CC}}$ is a coupling capacitor whereas $\mathrm{L}_{\mathrm{C}} \mathrm{C}_{\mathrm{C}}, \mathrm{R}_{\mathrm{S}}$ and $\mathrm{R}_{\mathrm{L}}$ are external components with the value of $0.1 \mu \mathrm{H}, 100 \mathrm{pF}, 50 \Omega$, and $50 \Omega$ respectively. The components used to stabilize the circuits are input resistor, RIN, input capacitor, $\mathrm{C}_{\mathrm{IN}}$, output resistor, ROUT, output capacitor, COUT, output resistor of second stage, ROUT2, and output capacitor of second stage, COUT2. On the other hand, the source resistor, RS, bypass capacitor, $\mathrm{CS}_{\mathrm{S}}$, source resistor of second stage, RS2, bypass capacitor of second stage, $\mathrm{C}_{\mathrm{S} 2}$, and input resistor of second stage, RIN2, are used to bias M1 and M2, ensuring $0 \mathrm{~V}$ at M1 and M2 gates. In addition, feedback resistor, RFB, feedback capacitor, CFB, feedback inductor, LFB, feedback resistor of second stage, RFB2, and feedback capacitor of second stage, CFB2 are used to feedback network for M1 and M2. All these components have significant effect on the MMIC LNA performance on power gain $(\mathrm{dB}(\mathrm{S}(2,1))$, noise figure (NFmin_out), drain current (ID), as well as circuit stability factors (mu_load \& mu_source). As a result, optimized combination of these components is desired for a better circuit design.

\section{Figure 1: The designed circuit for RC feedback amplifier}

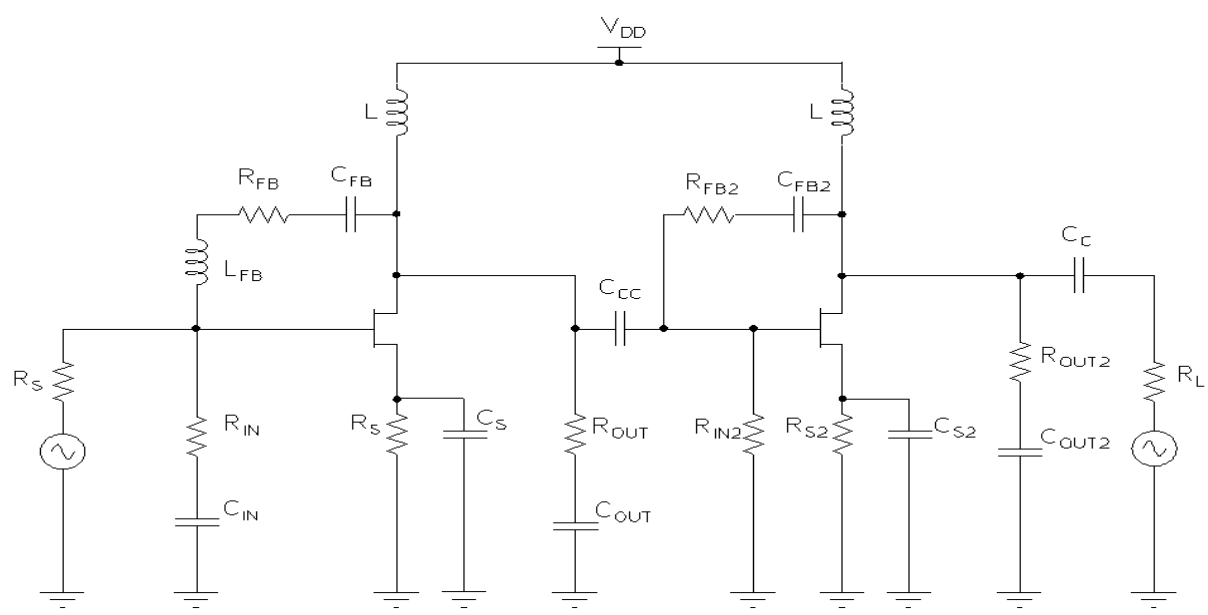

The allowable range of the design variables or components for the circuit is given in Table 1 whereas the intended outcome or specifications on circuit performance are given in Table 2 . From Table 2, multiple optimization aims are shown in which noise figure and drain current are to be minimized whereas power gain is to be maximized. The column of weight indicates the priority preference of optimization. 
Table 1: Setup of design variables

\begin{tabular}{lll}
\hline Design Variables & From & To \\
\hline RFB $(O h m)$ & 10 & 550 \\
Finger & 2 & 10 \\
CFB(pF) & 4 & 7 \\
ROUT(Ohm) & 100 & 300 \\
COUT(pF) & 2 & 20 \\
RIN(Ohm) & 500 & 1000 \\
CIN(pF) & 2 & 20 \\
LFB(nH) & 1 & 10 \\
CCC(pF) & 1 & 20 \\
RS(Ohm) & 5 & 50 \\
CS(pF) & 1 & 100 \\
RS2(Ohm) & 5 & 50 \\
\hline
\end{tabular}

Table 2: The Required Specifications for Amplifier Optimization

\begin{tabular}{lllll}
\hline Objective & \multicolumn{2}{c}{ Value Range } & Optimization & Weight \\
\cline { 2 - 3 } & \multicolumn{2}{c}{ Minimum } & Maximum & \\
\hline NFmin_out(dB) & ---- & 2.5 & Minimize & 5 \\
dB(S(2,1) & 17 & 20 & Maximize & 1 \\
mu_load & 1.05 & ---- & ---- & 1 \\
mu_source & 1.05 & ---- & ---- & 1 \\
ID $(A)$ & 0.04 & 0.05 & Minimize & 10 \\
\hline
\end{tabular}

\section{PSO FOR MMIC DESIGN}

PSO is a population based optimization algorithm, modeled after the social flocking behavior of birds. In PSO, each particle represents a solution to a problem and a swarm is composed of these particles. The problem solution space is formulated as search space while search space refers to the correlated solution of the problem (Bo \& Xia, 2009). For instance, in a $d$-dimensional search space, the position of the $i^{- \text {th }}$ particle, $p_{i}$, are mathematically represented as

$$
p_{i}=\left[p_{i 1}, p_{i 2}, p_{i 3}, \ldots, p_{i d}\right], \text { where } i=1,2, \ldots, n
$$

By refering to Table 1, a total of 12 design variables to be optimized indicate 12-dimensional search space to be explored. At the initialization state, 40 particles with 12 -dimensional design variable setting are generated. Each of these particles is then evaluated based on a fitness function which determines the performance of the design variables setting. Equation (2-6) show the fitness function evaluation for power gain $(\mathrm{dB}(\mathrm{S}(2,1))$, noise figure (NFmin_out), drain current (ID), as well as circuit stability factors (mu_load \& mu_source). A penalty value, $\alpha$ is given to the fitness function when the required constraints are not complied.

$$
\begin{aligned}
& F 1=\left\{\begin{array}{l}
\alpha+\left(\mathrm{NFmin} \_ \text {out }-2.5\right), \text { if NFmin_out }>2.5 \\
\alpha+(0.1-\mathrm{NF} \text { min_out }), \text { if NFmin_out }<0.1 \\
\text { NFmin_out- } 0.1, \text { if } 0.1<\mathrm{NFmin} \_ \text {out }<2.5
\end{array}\right. \\
& F 2=\left\{\begin{array}{l}
\alpha+(\mathrm{dB}(\mathrm{S}(2,1))-20), \text { if } \mathrm{dB}(\mathrm{S}(2,1))>20 \\
\alpha+(17-\mathrm{dB}(\mathrm{S}(2,1))), \text { if } \mathrm{dB}(\mathrm{S}(2,1))<17 \\
20-\mathrm{dB}(\mathrm{S}(2,1)), \text { if } 17<\mathrm{dB}(\mathrm{S}(2,1))<20
\end{array}\right.
\end{aligned}
$$




$$
\begin{gathered}
F 3=\left\{\begin{array}{l}
\alpha, \text { if mu_load }<1.05 \\
0, \text { if } \text { mu_load }>1.05
\end{array}\right. \\
F 4=\left\{\begin{array}{l}
\alpha, \text { if } \text { mu_source }<1.05 \\
0, \text { if } \mathrm{mu} \_ \text {source }>1.05
\end{array}\right. \\
F 5=\left\{\begin{array}{l}
\alpha+(0.04-\mathrm{ID}), \text { if } \mathrm{ID}<0.04 \\
\alpha+(\mathrm{ID}-0.05), \text { if } \mathrm{ID}>0.05 \\
\mathrm{ID}-0.04, \text { if } 0.04<\mathrm{ID}<0.05
\end{array}\right.
\end{gathered}
$$

This research applied a normalized weighted sum approach as proposed by Morad (1997) to obtain a total fitness evaluation, so called $F_{t o t}$, as given in Equation (7). The main reason for applying the normalized weighted sum approach is to reduce the possible bias caused by different fitness function that consist of different unit of evaluation.

$$
F_{\text {tot }}=5 \frac{F 1}{F \text { laverage }}+\frac{F 2}{F 2 \text { average }}+\frac{F 3}{F 3 \text { average }}+\frac{F 4}{F 4 \text { average }}+10 \frac{F 5}{F 5 \text { average }}
$$

In this research, the MMIC is optimized at a specified frequency of $5 \mathrm{GHz}$ by minimizing the $F$ tot value. In other words, the smaller the $F_{t o t}$, the better the fitness is. Different weight is given to the particular objective fitness based on the preference of the circuit designer. Based on the $F$ tot equation, fitness of each particle will be evaluated to identify the local best particle, $P_{\text {ibest }}$, and the global best particle, $P_{\text {gbest }}$ in the swarm. Based on the local and global fitness, each particle in the swarm will be assigned a velocity value, $V_{i}$, that will affect the step movement of the particle in the problem search space where

$V_{i}=\left[v_{i 1}, v_{i 2}, v_{i 3}, \ldots . ., v_{i d}\right] \quad$, where $i=1,2, \ldots, n$

In PSO, each particle is flown through the multidimensional search space, adjusting its position according to its memory on Pibest and Pgbest in the iterations encountered. Moving toward the optimal solution, the fitness of all particles in the neibourhood is considered to adjust the particles' velocities for updating the particles' positions. Equation (9) and (10) show respectively the adjustment of particle's velocity and position based on Pibest and Pgbest.

Let $t$ be the current iteration number, $j$ be the particular dimension of an $i^{-t h}$ particle, and $w$ be the inertia factor of the particle. $c_{1}$ and $c_{2}$ represent the cognitive and social factor respectively whereas $r_{1}$ and $r 2$ represent the uniformly distributed random number from the interval of 0 to 1 .

$v_{i j}(t+1)=w v_{i j}(t)+c_{1} r_{1}\left[\left(P_{i b e s t j}(t)-P_{i j}(t)\right]+c_{2} r_{2}\left[\left(P_{\text {gbestj }}(t)-P_{i j}(t)\right]\right.\right.$

$=P_{i j}(t)+v_{i j}(t+1)$, where $j=1,2, \ldots, d$

The step by step mechanism of PSO is shown below, more details can be obtained from Dong and Qiu (2006):

Step 1: Define the value for inertia, cognitive and social factor. Then, randomly initialize paticle positions and the respective velocities.

Step 2: Fitness of each particle is evaluated using equation (7).

Step 3: The fitness of each particle, $P_{i}$, is compared with the local best particle, $P_{i b e s t}$. If the fitness value is better than the $P_{i b e s t}$ from the previous iterations , then $P_{i}$ is taken as the local best particle, $P_{i b e s t}$.

Step 4: The fitness of each particle, $P_{i}$, is compared with the global best particle, $P_{g}$ best. If the fitness value is better than the Pgbest from the previous iterations, then $P_{i}$ will replace the global best particle, Pgbest.

Step 5: Each particle's velocity for the next iteration is calculated using equation (9). Based on the updated velocity, each particle's position is updated using equation (10). Step 6: Repeat step 2 to step 5 until the termination criterion is met. This research implements PSO on a 5GHz MMIC LNA. First, a PSO model is developed to find the design variable setting for the MMIC LNA. Then the circuit performance for each design setting is simulated using the ADS. The result obtained from ADS is fed back to the PSO model for optimization purpose. The process of simulation and optimization continue until a termination criterion is met. The PSO mechanism is terminated when the required specification and the performance for the MMIC design is achieved.

\section{Result and Discussion}

In this research, 40 particles are generated and the PSO mechanism terminates in the $5^{\text {th }}$ iteration where the required specifications and the performance of the MMIC design is fulfilled. In the developed PSO, 
the decreasing inertia weight, $w$, is set from 0.4 to 0.9 whereas the cognitive and social factor, $c_{1}$ and $c_{2}$ are both set to 2 . Based on the result obtained, the optimized design variables proposed by PSO in the $5^{\text {th }}$ iteration is given in Table 3. The achievements of objectives such as power gain $(\mathrm{dB}(\mathrm{S}(2,1))$, noise figure (NFmin_out), drain current (ID), as well as circuit stability factors (mu_load \& mu_source) of the optimized MMIC design variables are shown in Table 4.

Table 3: Optimized Design Variables For MMIC Amplifier Using PSO

\begin{tabular}{ll}
\hline Design Variable & Value \\
\hline RFB(Ohm) & 113.867 \\
Finger & 10 \\
CFB(pF) & 6.922 \\
ROUT(Ohm) & 175.177 \\
COUT(pF) & 9.19 \\
RIN(Ohm) & 663.95 \\
CIN(pF) & 7.074 \\
LFB(nH) & 10 \\
CCC(pF) & 8.356 \\
RS(Ohm) & 36.302 \\
CS(pF) & 10.97 \\
RS2(Ohm) & 40.429 \\
\hline
\end{tabular}

Table 4 shows the comparison of different optimizer in optimizing the 5GHz MMIC design. Conventional trial and error approach is not fulfilling the specification in terms of $\operatorname{ID}$ and $\operatorname{dB}(\mathrm{S}(2,1)$. Besides, it is widely known that trial and error approach is not systematic and might be time consuming. In other hand, a number of built-in optimizer from the ADS such as Gradient, Random Minimax, and Quasi-Newton are used to optimize the designed MMIC. From the results, it is observed that these built-in optimizers fulfill the specification requirements. However, the produced ID value is high which is very near to the upper boundary of the required specification. Based on the weight given to the prefered objective, ID is the most prefered as the weight factor is given as 10 as compared to NFmin_out which is given as 5 , follow by $\mathrm{dB}(\mathrm{S}(2,1)$ and the two stability factors which are all given as 1 . Although all built-in optimizers produce very good $\mathrm{dB}(\mathrm{S}(2,1)$, the achievement for ID and NFmin_out are not satisfactory due to the weight given as compared to the proposed PSO. Eventhough PSO produce slightly less $\mathrm{dB}(\mathrm{S}(2,1)$, it manage to generate a design which could complement the ID and NFmin_out which are of more importance. From Table 4, PSO gives the smallest ID and NFmin_out which are 0.0467 and 1.1533 respectively. In overall performance, PSO is shown to be a better alternative as compared to the other built-in optimizers as well as the conventional trial and error approach.

Table 4: Comparison of Different Optimizers

\begin{tabular}{llllll}
\hline & Nfmin_out & dB(S(2,1)) & mu_load & \multicolumn{2}{l}{ mu_sourceID } \\
\hline Trial and Error & 1.98834 & 20.7525 & 4.66684 & 2.38164 & 0.05143 \\
Gradient & 2.116 & 19.991 & 4.999 & 2.346 & 0.0499619 \\
RandomMinimax & 2.221 & 19.893 & 5.028 & 2.014 & 0.0493366 \\
Quasi-Newton & 2.167 & 19.617 & 5.19 & 2.327 & 0.0493913 \\
PSO & 1.1533 & 19.7379 & 1.7475 & 1.2174 & 0.0467 \\
\hline
\end{tabular}

\section{Conclusion}

In this research, PSO is used to optimize the 5GHz MMIC LNA design. By implementing the normalized weighted-sum approach and considering the particle feasibility by using the constraint 
equations, the developed PSO is shown to be performing well in fulfilling the specified requirement and optimizing a number of objectives. The objectives for designing the LNA include power gain, noise figure, drain current and two circuit stability factors. The developed PSO is compared to a number of built-in optimizer from the ADS. Based on the weight preference given by the LNA designer, PSO optimize the MMIC LNA design accordingly. In overall performance, the developed PSO is shown to be a better alternative as compared to the ADS built-in optimizers such as Random Minimax, Gradient, and Quasi Newton. In terms of comparison to the generally time consuming conventional trial and error approach, PSO is a more systematic method for assisting the optimization of the MMIC LNA design.

\section{References}

Bo, L. \& Xia, P. H. (2009). A Hybrid PSO-DV Based Intelligent Method for Fault Diagnosis of Gear Box. Proceedings of the IEEE international Symposium on CIRA, 451-456.

Dong, C. J. \& Qiu, Z. L. (2006). Particle Swarm optimization Algorithm Based on the Ideal Simulating Annealing. International Journal of Computer Science and network Security, 6, 152-156.

Gatta, F., Sacchi, E., Suelto, F., Vilmercati, P. \& Castello, R. (2001). A2-dB noise figure 900-MHz differential CMOS LNA. IEEE J. Solid-State Circuits, 36, 1444-1452.

Ge, J. Y. \& Mayaram, K. (1998). A Comparative Analysis of CMOS Low Noise Amplifiers for RF Applications. ISCAS, 4, 349-352.

Gil, J., Han, K. \& Shin, H. (2003). 13 GHz 4.67 dB NF CMOS low noise amplifier. Elec. Letters, 39, 10561058.

Gupta, R. \& Allstot, D. J. (1998). Parasitic-Aware Design and d to Optimizationof CMOS RF Integrated Circuits. IEEE MTT-S Int.Microwave Symposium, 3, 1867-1870.

Kennedy, J. \& Eberhart, R. (1995). Particle swarm optimization. Proceedings of the IEEE International Conference on Neural Networks, 4, 1942-1948.

Lucek, J. \& Damen, R. (1999). LNA Design for CDMA front end. RF Design, Intertec.

Marsh, S. (2006). Practical MMIC Design, Artech House.

Morad, N. (1997). Optimization of cellular manufacturing systems using genetic algorithms. Ph.D. dissertation, Univ. Sheffield, Sheffield, U.K., 1997.

Neoh, S. C., Marzuki, A., Morad, N., Lim, C. P.\& Abdul- Aziz, Z. (2008). An Interactive Evolutionary Algorithm to MMIC Low Noise Amplifier Design. Innovative Computing, Information, and Control Express Letter, ICIC-EL.

Niknejad, A. M. \& Meyer, R. G. (1998). Analysis, Design and Optimizationof Spiral Inductors and Transformers for Si RF ICs. IEEE J. Solid-State Circuits, 33, 1470-1481.

Nocedal, J. \& Wright, S. J. (1999). Numerical Optimization, Springer-Verlag, New York, 1999.

Robertson, I. D. \& Lucyszyn, S. (2001). RFIC and MMIC Design and Technology, IET Circuits, Devices, and System Series 13.

Tulunay, G. \& Balkir, S. (2004). A Compact Optimization Methodology For Single Ended LNA. IEEE International Symposium on Circuits and Systems, 273-276.

Taris, T., Begueret, J. B., Lapuyade, H. \& Deval, Y. (2003). A 1-V2GHz VLSI CMOS Low Noise Amplifier. RFIC Symposium, 123-126.

Vancorenland, P., Ranter, C. D., Steyaert, M. \& Gielen, G. (2000). Optimal RF Design using Smart Evolutionary Algorithms. Design Automation Conf. 2000, 7-10.

Yang, J. T., Fan, H. P., Tsai, P. J. \& Wu, M. J. (2009). A RF CMOS Low Noise Amplifier for WiMAX Applications. IEEE ISCIT 2009. 